\title{
Glatiramer Acetate Attenuates Pro-Inflammatory T Cell Responses but Does Not Directly Protect Neurons from Inflammatory Cell Death
}

\author{
Alexander M. Herrmann, ${ }^{*}$ Kerstin Göbel, ${ }^{*}$ \\ Ole J. Simon, ${ }^{\dagger}$ Nico Melzer, ${ }^{* \dagger}$ \\ Michael K. Schuhmann, ${ }^{*}$ Max-Philipp Stenner, ${ }^{\dagger}$ \\ Andreas Weishaupt, ${ }^{\dagger}$ Christoph Kleinschnitz, ${ }^{\dagger}$ \\ Stefan Bittner, ${ }^{\star \dagger}$ Patrick Meuth, ${ }^{\ddagger}$ Olaf Stuve, ${ }^{\S}$ \\ Thomas Budde, ${ }^{\ddagger}$ Bernd C. Kieseier, ${ }^{\text {Tा }}$ \\ Heinz Wiendl, ${ }^{*}$ and Sven G. Meuth ${ }^{\star \neq}$
}

Glatiramer acetate (GA) is a synthetic, random, basic copolymer capable of modulating adaptive $T$ cell responses. In animal models of various inflammatory and degenerative central nervous system disorders, GA-induced $T$ cells cross the blood-brain barrier, secrete high levels of anti-inflammatory cytokines and neurotrophins, and thus both reduce neuronal damage and promote neurogenesis. Recently, it has been suggested that GA itself may permeate the (impaired) blood-brain-barrier and directly protect neurons under conditions of inflammation-mediated neurodegeneration. To test this hypothesis, we examined the direct effects of GA on neuronal functionality and $\mathbf{T}$ cell-mediated neuronal apoptosis in culture, acute brain slices, and focal experimental autoimmune encephalomyelitis. GA caused a depolarization of the resting membrane potential and led to an immediate impairment of action potential generation in neurons. Moreover, GA-incubated neurons underwent dose-dependent apoptosis. Apoptosis of ovalbumin peptide-loaded major histocompatibility complex class I-expressing neurons induced by ovalbumin-specific effector $T$ cells could be reduced by pre-incubation of $T$ cells, but not neurons with GA. Similar results could be found using acute brain slices. In focal experimental autoimmune encephalomyelitis, lesion size and neuronal apoptosis could be limited by pretreating rats with GA, whereas intracerebral GA application into the inflammatory lesion had no effect on neuronal survival. Our data suggest that GA attenuates adaptive pro-inflammatory $T$ cell responses, but does not exert direct neuroprotective effects. (Am J Pathol 2010, 177:3051-3060; DOI: 10.2353/ajpath.2010.100442)

Glatiramer acetate (GA), is a synthetic random basic copolymer composed of tyrosine, glutamic acid, alanine, and lysine, capable of modulating adaptive $T$ cell responses. After binding to major histocompatibility complex (MHC) class II molecules (MHC II) on antigen-presenting cells (APCs), GA may act as a partial agonist and shift adaptive $T$ cell responses from a pro-inflammatory $T$ helper cell $(\mathrm{Th})_{1}$ toward an anti-inflammatory $\mathrm{Th}_{2 / 3}$ pattern of cytokine secretion. ${ }^{1-3}$ Moreover, independent form binding to MHC II molecules, GA may induce the development of APCs that secrete an anti-inflammatory "type II" cytokine pattern and thus promote the differentiation of naive $\mathrm{T}$ cells into $\mathrm{Th}_{2 / 3}$ and regulatory $\mathrm{T}$ cells. ${ }^{4}$

In different animal models of inflammatory ${ }^{5-7}$ and degenerative ${ }^{8-10} \mathrm{CNS}$ disorders, it was shown that peripheral GA-induced $T$ cells cross the blood-brain barrier, secrete high levels of anti-inflammatory cytokines and various neurotrophins, and thereby indirectly reduce neu-

Supported by the Federal Ministry of Education and Research, Germany, (BMBF), the Clinical Competence Network Multiple Sclerosis (KKNMS) and its subconsortium (UNDERSTAND MS), as well as by university grants (IZKF Z-3/4) to N.M. and H.W. and (IZKF A54-1) to S.G.M. and H.W. Furthermore we thank Teva for financial support and discussion.

A.M.H., K.G., O.J.S., N.M., H.W., and S.G.M contributed equally

Accepted for publication August 3, 2010

All authors declare no conflict of interest.

Supplemental material for this article can be found on http://ajp. amjpathol.org.

Address reprint requests to Sven Meuth, M.D., Ph.D., or Heinz Wiendl, M.D., Department of Neurology - Inflammatory Disorders of the Nervous System and Neurooncology, University of Münster, Domagkstr. 13, 48149 Münster, Germany. E-mail: sven.meuth@ukmuenster.de or heinz.wiendl@ ukmuenster.de. 
ronal and axonal damage. ${ }^{5,9,11,12}$ These effects of GAinduced $T$ cells are amplified by a profound bystander effect on microglia and astrocytes, leading to an increased expression of neurotrophins at the site of injury and a reduced release of glutamate, nitric oxide, and pro-inflammatory cytokines by these cells. ${ }^{8-10,13}$ Additionally, following peripheral application, GA-induced T cells may also promote neurogenesis by promoting proliferation of neural progenitor cells, their migration toward lesioned sites and differentiation into mature neurons. ${ }^{14}$

Under certain conditions of inflammation-related neurodegeneration GA-induced neuroprotective effects were observed before an adaptive immune response was established, ${ }^{10}$ suggesting that GA-induced neuroprotection may be partially $T$ cell-independent. Indeed, it has been suggested that GA may permeate the (impaired) blood-brain barrier either per se or via uptake and release by CNS-invasive immune cells and therefore directly protect neurons/axons under conditions of inflammation-related neurodegeneration. ${ }^{15}$ However, it remains unclear at present how such a protective effect is mediated directly. Therefore, we studied the effects of GA on electrical properties of neurons and $T$ cell-mediated neuronal apoptosis in culture, acute brain slices, and focal experimental autoimmune encephalomyelitis (EAE).

\section{Materials and Methods}

\section{Animals}

Wilde type (WT) C57BL/6, B2-microglobulin ${ }^{-1-16}$, transgenic mice expressing ovalbumin (OVA) in oligodendrocytes (ODCs) (ODC-OVA), ${ }^{17}$ and mice transgenic for a T cell receptor recognizing the ovalbumine $257-264$ (OVA) peptide in the context of $\mathrm{H}_{2} \mathrm{~K}^{\mathrm{b}} \mathrm{MHC}-\mathrm{I}$ molecules $(\mathrm{OT}-\mathrm{I})^{18}$ and Dark Agouti (DA) rats were kept under pathogen-free conditions and had access to food and water ad libitum. All experiments were conducted according to the German law of animal protection and were approved by local authorities.

\section{OT-I Isolation, Culture, and Stimulation}

Isolation of $\mathrm{CD}^{+}$OT-I T cells (recognizing the ovalbu$\mathrm{min}_{257-264}$ peptide [OVA] in the context of $\mathrm{H} 2-\mathrm{K}^{\mathrm{b}} \mathrm{MHC}-\mathrm{I}$ molecules) and generation of effector $T$ cells was performed as previously described. ${ }^{19,20}$ In a subset of experiments, incubation of OT-I T cells with OVA was performed in the presence of various concentrations of GA $(1,20$, and $100 \mu \mathrm{g} / \mathrm{ml}$ GA provided by Teva Pharmaceuticals, Petach Tikva, Israel) prepared in splenocytescomplete medium for 5 days.

Before and after 5 days of in vitro culture, flow cytometry of splenocytes $\left(1 \times 10^{6}\right.$ cells $)$ was routinely performed using standard methods. ${ }^{19}$ For analysis of $\mathrm{T}$ cell subtype distribution, cells were stained for 20 minutes with phycoerythrin-labeled anti-mouse CD8 and fluorescein isothiocyanate-labeled anti-mouse CD4 antibodies (all BD Bioscience, Heidelberg, Germany). As isotype controls, splenocytes were stained either with phycoerythrin-labeled anti-mouse IgG1 or fluorescein isothiocyanate-labeled anti-mouse IgG1 (all BD Bioscience,
Heidelberg, Germany). After 5 days of in vitro stimulation with OVA, OT-I splenocytes consist of 95 to $99 \% \mathrm{CD}^{+}$ cells. For analysis of T cell activation markers, cells were stained for 20 minutes with fluorescein isothiocyanatelabeled anti-mouse CD44, allophycocyanin-labeled antimouse CD62L and phycoerythrin-labeled anti-mouse CD11a (all BD Bioscience, Heidelberg, Germany). All antibodies were titrated for optimal staining. Flow cytometry analysis was performed using a FACS Calibur system (BD Biosciences, Heidelberg, Germany) and results were analyzed using the FlowJo Software (Tree Star, Ashland, OR).

For proliferation analysis of OVA stimulated OT-I, cells were incubated with $10 \mu \mathrm{mol} / \mathrm{L}$ carboxyfluorescein diacetate succinimidyl ester (Molecular Probes, Karlsruhe, Germany). After 5 days of in vitro culture, OT-I T cells were collected and their proliferative responses were detected based on carboxyfluorescein diacetate succinimidyl ester division by flowcytometry performed on a FACS Calibur (BD Bioscience, Heidelberg, Germany).

\section{Hippocampal Neuronal Cell Culture and Co-Culture Experiments with OT-I T Cells}

Neuronal cell cultures were obtained from WT C57BL/6 mice embryos (E18) following previously described protocols. ${ }^{21,22}$ Neuronal cultures were incubated at $37.0^{\circ} \mathrm{C}$ and $5 \% \mathrm{CO}_{2}$ and held in culture for up to 5 to 7 days before experiments. Immunocytochemical staining (see below) for nuclei (4,6-diamidino-2-phenylindole [DAPI]), neurons (Microtubule associated protein 2a; MAP2a), and astrocytes (glial fibrillary acidic protein) provided a purity of $\sim 80 \%$ of our neuronal cell culture system.

For OT-I cytotoxicity assays, hippocampal cell cultures were incubated for 48 hours with pure neuronal medium or neuronal medium containing $500 \mathrm{U} / \mathrm{ml}$ interferon- $\gamma$ (Pepro Tech, London, UK). Neurons were loaded with OVA (100 nmol/L) and subsequently in vitro activated OT-I T cells were added at a density of 60,000 cells $/ \mathrm{cm}^{2}$ and co-incubated for 6 hours. To analyze cell death following OT-I T cell-neuron interactions, co-cultures were fixed with 4\% paraformaldehyde (Merck, Darmstadt, Germany), washed three times with $10 \mathrm{mmol} / \mathrm{L}$ PBS and incubated for 90 minutes at $4{ }^{\circ} \mathrm{C}$ in $10 \mathrm{mmol} / \mathrm{L}$ PBS containing 10\% horse normal serum (PAA Laboratories, Pasching, Austria) and 0.2\% Triton X100 (Sigma, Munich, Germany). Subsequently, primary antibodies (NeuN 1:1000, Chemicon, Billerica, MA, and activated caspase-3, 1:200, Cell Signaling, Boston, MA) were diluted in 10 $\mathrm{mmol} / \mathrm{L}$ PBS containing $1 \%$ horse normal serum and incubated for 1 hour at room temperature. After washing steps with $10 \mathrm{mmol} / \mathrm{L}$ PBS, secondary antibodies (Cy-2 labeled donkey anti-mouse 1:100, Cy-3 labeled goat anti-rat 1:100, both from Dianova, Hamburg, Germany) were incubated in the same manner. Staining with $0.5 \mu \mathrm{g} / \mathrm{ml}$ DAPI (Merck, Darmstadt, Germany) was performed for 5 minutes. Finally, cultures were washed and subsequently covered with 1,4diazobicyclo-2,2,2-octane (DABCO; Merck, Darmstadt, Germany) and the fraction of activated caspase-3-positive neurons was determined by immunofluorescence microscopy (Axiophot; Zeiss, Jena, Germany). 
Whole Cell Patch Clamp Recordings of Hippocampal Neurons from WT and B2-Microglobulin-Deficient Mice

Whole-cell patch clamp recordings were performed on cultured hippocampal neurons at room temperature (21 to $23^{\circ} \mathrm{C}$ ) as described earlier. ${ }^{22}$ For a subset of experiments, GA (100 $\mu \mathrm{g} / \mathrm{ml})$ and mannitol $(200 \mathrm{mg} / \mathrm{ml})$ were added to the extracellular solution before adjustment of $\mathrm{pH}$ and osmolality. All neurons included in the analysis had a resting membrane potential negative to $-50 \mathrm{mV}$, the access resistance was typically in the range of 5 to 15 $\mathrm{M} \Omega$ and series resistance compensation of more than $40 \%$ was routinely used. A liquid junction potential of $8 \pm$ $2 \mathrm{mV}(n=10)$ was measured and taken into account when analyzing the data. The resting membrane potential and action potential discharge behavior of neurons were determined using the current clamp method and depolarizing step protocols.

\section{Extracellular Recording of Neuronal Network Activity during T Cell-Neuron Interactions Using Multi-Electrode Arrays}

To analyze the impact of GA on the activity of neuronal networks, different parameters of neuronal network activity were determined using the multi-electrode array technique $^{22,23}$ in the absence and the presence of GA at a concentration of $10 \mu \mathrm{g} / \mathrm{ml}$. Multi-electrode array detects compound field potentials in the spatial vicinity of multiple extracellular electrodes in densely cultured hippocampal neurons, which are capable of generating spontaneous synchronized activity. Rat hippocampal neurons (QBM Cell Science, Ottawa, Canada) were plated at a density of $2 \times 10^{5} / \mathrm{cm}^{2}$ on multi-electrode array chips. Multielectrode array chips contained 60 Ti/TiN microelectrodes (Multi-Channel Systems, Reutlingen, Germany) each with an input resistance of $<50 \mathrm{k} \Omega$, a diameter of 30 $\mu \mathrm{m}$ and a mean distance to the neighboring electrode of $200 \mu \mathrm{m}$. All electrode signals were sampled at a frequency of $25 \mathrm{kHz}$ using the MC-Rack Standard software (Multi-Channel Systems, Reutlingen, Germany). During the incubation we analyzed the relative number of active channels and the frequency of compound field potentials detected by single channels as a measure of neuronal network activity.

\section{Preparation of Acute Brain Slices and Co-Culture Experiments with OT-I T Cells}

Preparation of acute brain slices was performed following standard procedures like described before. ${ }^{19,24}$ For experiments, slices were incubated with or without $5 \times 10^{5}$ OT-I T cells in the absence or the presence of various concentrations of GA $(10,50,100 \mu \mathrm{g} / \mathrm{ml})$ with standard artificial cerebrospinal fluid (ACSF) (mmol/L: $\mathrm{NaCl}, 125$; $\mathrm{KCl}, 2.5 ; \mathrm{NaH}_{2} \mathrm{PO}_{4}, 1.25 ; \mathrm{NaHCO}_{3}, 24 ; \mathrm{MgSO}_{4}, 2 ; \mathrm{CaCl}_{2}$, 2; dextrose, 10; $\mathrm{pH}$ adjusted to 7.35 by bubbling with a mixture of $95 \% \mathrm{O}_{2}$ and $5 \% \mathrm{CO}_{2}$ ) for 6 hours. Afterward slices were harvested and embedded using optimal cutting temperature compound (Tissue-Tek, Sakura Finetek Europe, Zoeterwoude, Netherlands) and frozen in liquid nitrogen. Ten-micron coronary cryosections were obtained using a cryostat (Leica CM 1950, Wetzlar, Germany).

\section{Induction of Focal EAE in DA Rats}

To induce focal $\mathrm{EAE}^{25}$ recombinant myelin oligodendrocyte glycoprotein corresponding to the N-terminal sequence of rat myelin oligodendrocyte glycoprotein was expressed in Escherichia coli and purified as described previously. ${ }^{26}$

DA rats were actively immunized with $85 \mu \mathrm{g} \mathrm{MOG}$ (diluted in saline) emulsified in complete Freund's adjuvant (CFA, Sigma-Aldrich, Munich, Germany). Rats were evaluated on a daily basis for changes in body weight, overt signs of illness, and clinical signs of EAE by two blinded investigators using the following 5-point scoring system: 0, no neurological signs; 1, tail limpness; 2 , broad-based gait and pronounced ataxia; 3, hind limb paraparesis; 4, tetraparesis; 5, death attributable to EAE.

Twenty-four hours after symptom onset, immunized rats were anesthetized with isoflurane, placed in a stereotactic frame and a fine hole was drilled through the scull giving access to the brain $1 \mathrm{~mm}$ caudal to the bregma and $2 \mathrm{~mm}$ lateral to the sagittal suture. Rats were injected with $4 \mu \mathrm{l}$ of a mixture of pro-inflammatory cytokines (250 ng TNF $\alpha$ and $100 \cup$ interferon- $\gamma$; both Peprotech London, UK) dissolved in PBS. GA (40 $\mu \mathrm{g})$ or PBS alone was injected into the dorsal lateral geniculate nucleus of the thalamus (dLGN). A trace of Chicago blue (Sigma-Aldrich, Munich, Germany) was added as a marker dye for better visibility. Postsurgical recovery was uneventful in all cases with no additional clinical signs.

Two days after intracerebral (i.c.) injection rats were anesthetized with isoflurane. The brain was removed and embedded in optimal cutting temperature compound. Ten-micron coronary cryosections were obtained using a cryostat.

\section{Immunofluorescence Staining and Confocal Microscopy}

Immunofluorescence-staining was performed as described before. ${ }^{19}$ Antibodies used in this study were antiNeuN (1:1000, Chemicon), anti-NogoA (1:750, Chemicon), anti-activated caspase-3 (1:200, Cell Signaling), Alexa Fluor 488-coupled goat antibodies recognizing mouse IgG (1:200, Invitrogen, Karlsruhe, Germany) and Cy3-coupled goat antibodies recognizing rabbit IgG (1: 300, Dianova, Hamburg, Germany). For detection of apoptosis, a terminal deoxynucleotidyl transferase dUTP nick-end labeling (TUNEL) in situ Cell Death Detection Kit (Roche, Basel, Switzerland) was used according to the manufacturer's instructions. Negative controls were obtained by either omitting the primary or secondary antibody and revealed no detectable signal on subsequent analysis. 
A
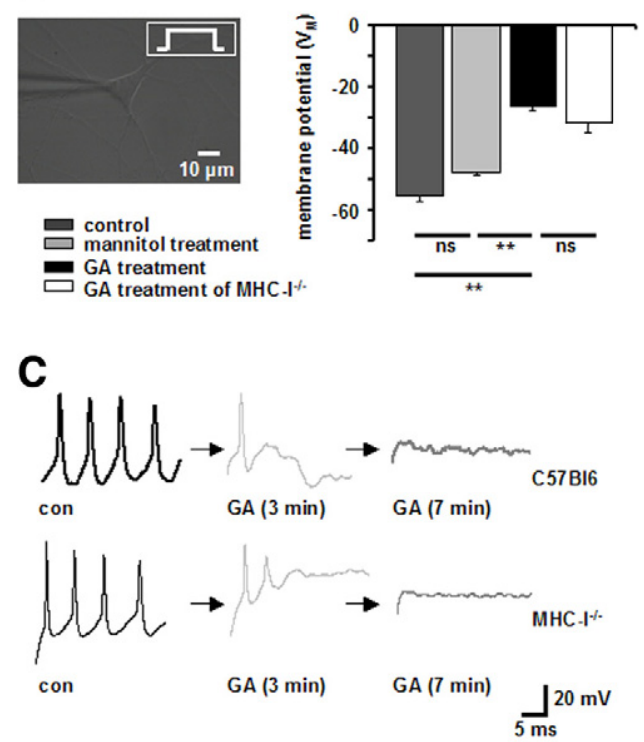

B

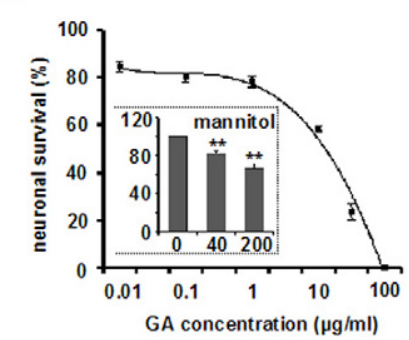

D

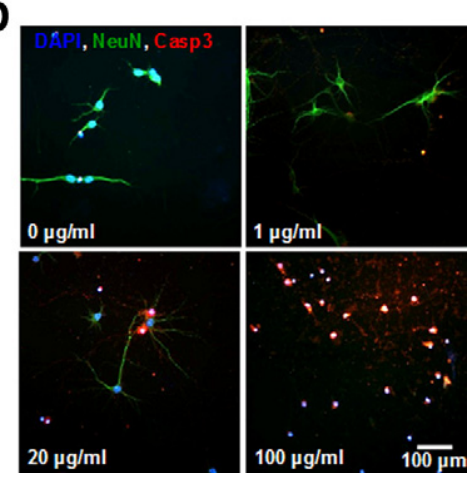

Figure 1. A and C: GA causes a depolarization of the resting membrane potential and an immediate impairment of action potential generation in cultured hippocampal neurons. A: Resting membrane potential of cultured WT and MHC-I-deficient hippocampal neurons in the absence or presence of GA $(100 \mu \mathrm{g} / \mathrm{ml}, n=5$, respectively $)$ and its solvent mannitol ( $200 \mu \mathrm{g} / \mathrm{ml}, n=3)$ as determined by whole-cell patch clamp recording. C: Representative recordings of action potential firing in cultured WT (upper panels) and MHC-I-deficient hippocampal neurons without GA (con), 3 and 7 minutes after incubation with GA $(100 \mu \mathrm{g} / \mathrm{ml})$. Black arrows indicate the time intercept between current traces. ns $=$ not significant, ${ }^{* *} P<0.05$. B and D: GA-incubated hippocampal neurons undergo dose-dependent apoptosis within 6 hours in culture. B: GA concentration-dependent fraction of activated caspase- $3^{+} \mathrm{NeuN}^{+}$hippocampal neuronal cell bodies in culture after 6 hours of incubation. (inset) Fraction of activated caspase- $3^{+}$ $\mathrm{NeuN}^{+}$hippocampal neuronal cell bodies in culture after 6 hours of incubation with the GA solvent mannitol at different concentrations $(0,40,200 \mu \mathrm{g} / \mathrm{ml})$, ns $=$ not significant, ${ }^{* * *} P<0.05$. D: Representative immunofluorescence images of cultured hippocampal neurons exposed for 6 hours to various GA concentrations. DAPI (blue), NeuN (green), activated caspase-3 (red). Scale bar $=100 \mu \mathrm{m}$.
Infiltrating cells were analyzed using antibodies against B115 (1:500, Holland Biotechnology, Leiden, Netherlands) or CD68 (ED-1, Linaris, Wertheim, Germany). Sections were then sequentially incubated with horse anti-mouse IgGbiotin (Vector Laboratories, Burlingame, CA), PBS, StreptABComplex (Dako, Glostrup, Denmark), PBS, and diaminobenzidine (Kem-En-Tec, Taastrup, Denmark).

For quantification of cell densities and lesion size, sections were examined using an Axiophot2 microscope (Zeiss, Oberkochen, Germany) equipped with a CCD camera (Visitron Systems, Tuchheim, Germany). Cell densities and lesion size were determined within preselected fields at specific sites (dentate gyrus of the hippocampus and piriform cortex for acute brain slices, dLGN for focal EAE) using MetaVue Software (Molecular Devices, Downingtown, PA).

\section{Statistical Analysis}

All results are presented as mean \pm SEM. Statistical analysis was performed using one-way analysis of variance with Bonferroni post hoc tests for group comparisons and Student's t-test modified for small samples ${ }^{27}$ for comparisons of two samples. $P$ values $<0.05$ were considered significant (indicated as ** in figures).

\section{Results}

\section{GA Causes a Depolarization of the Resting Membrane Potential and an Immediate Impairment of Action Potential Generation in Cultured Hippocampal Neurons}

To test the impact of the GA peptide on the electrical properties of neurons, cultured murine hippocampal neurons were patch-clamped and then incubated with GA at a concentration of $100 \mu \mathrm{g} / \mathrm{ml}$ for up to 10 minutes. The resting membrane potential and the electrical discharge behavior were determined via whole-cell patch clamp recording. Under control conditions, neurons exhibited a resting membrane potential after 10 minutes of recording of $-55.3 \pm 2.1 \mathrm{mV}(n=15)$, which was not significantly altered in the presence of the GA solvent mannitol (200 $\mu \mathrm{g} / \mathrm{ml} ;-47.6 \pm 2.3 \mathrm{mV} ; P=0.6 ; n=4)$. In contrast, addition of GA elicited a depolarization of the neuronal membrane potential to a steady state value of $-26.6 \pm$ $1.3 \mathrm{mV}(\mathrm{GA} 100 \mu \mathrm{g} / \mathrm{ml} ; P=0.0008 ; n=4)$ after 10 minutes (Figure 1A). Consistent with depolarization of the membrane potential, GA caused an immediate cessation of action potential generation elicited by applying a constant rectangular current pulse of 100 to $200 \mathrm{~mA}$ after about 3 minutes of incubation (Figure 1C, top panel). The immediate alteration of neuronal excitability by GA is not mediated by its binding to neuronal $\mathrm{MHC}$ I molecules, as both the depolarization of the membrane potential $(-31.75 \pm 3 \mathrm{mV} ; P=0.38 ; n=4$; Figure $1 \mathrm{~A})$ and the associated impairment of action potential generation (Figure 1C, bottom panel) were also observed in hippocampal neurons of MHC-I-deficient mice. Notably, the altered electrophysiological properties of neurons exposed to GA were accompanied by swelling of the soma, retraction of neurites, and membrane blebbing (data not shown), consistent with apoptosis. ${ }^{22}$ Hence, at concentrations of GA supposed to prevent neuronal cell death under conditions of oxidative stress, ${ }^{15}$ we observed an immediate impairment of neuronal excitability and morphological changes consistent with imminent apoptotic cell death.

Given the impairment of excitability of single hippocampal neurons by GA, we assessed its impact on neuronal network activity using the multi-electrode recording technique $^{22}$ in densely cultured hippocampal neurons (Supplemental Figure S1 at http://ajp.amjpathol.org). During the in- 
cubation period, the relative number of active channels and the relative frequency of compound field potentials of single channels were analyzed as a measure of neuronal network activity. As GA already induced an immediate depolarization of the membrane potential at a concentration of $100 \mu \mathrm{g} / \mathrm{ml}$ within minutes, the amount of GA was reduced to $10 \mu \mathrm{g} / \mathrm{ml}$ to analyze its effects in a functional neuronal network for a longer time period. The relative total number of active channels did not change significantly (Supplemental Figure S1, A and B, at http://ajp.amjpathol.org). However, in the presence of GA incubation we could detect firing frequencies elevated up to ten times in single channels as compared to its absence (Supplemental Figure S1C at http://ajp.amjpathol.org). These increased discharge frequencies could be elicited again on re-incubation with GA after periods of 15 hours without GA exposition.

Taken together, these data indicate that GA-induced neuronal membrane depolarization causing electrical silencing in single neurons can be gradual on the level of neuronal network activity and thus increases neuronal firing frequencies. Moreover, constant (or increased) neuronal network activity during the first 100 minutes after GA-incubation clearly demonstrates functional integrity of the neurons.

\section{GA Incubated Hippocampal Neurons Undergo Dose-Dependent Apoptosis within 6 Hours in Culture}

GA elicited neuronal membrane depolarization (although gradual), probably activates a variety of transmembrane transport processes, disturbs neuronal volume regulation, and elicits intracellular calcium accumulation via influx from the extracellular space and release from intracellular stores. When present for extended periods of time these cellular changes will result in neuronal cell death. Hence, we assessed the induction of apoptotic neuronal cell death by GA and its solvent mannitol after 6 hours of incubation in cultured hippocampal neurons by immunochemical analysis for activated caspase-3. Incubation for 6 hours with the GA-solvent mannitol alone resulted in significantly more apoptotic neurons compared to control conditions (Figure 1B, inset). Furthermore GA incubation (at a constant mannitol concentration) for 6 hours dose-dependently caused apoptosis in hippocampal neurons (Figure 1, $\mathrm{B}$ and $\mathrm{D}$ ) at a concentration supposed to directly protect neurons under conditions of oxidative stress. ${ }^{15}$

\section{Apoptosis of OVA Peptide-Loaded MHC-I-Expressing Neurons Induced by OVA-Specific OT-I Effector T Cells Is Reduced by Pre-Incubation of OT-I Splenocytes but Not Neurons with GA}

To investigate whether GA could prevent neuronal apoptosis resulting from direct $\mathrm{CD} 8^{+} \mathrm{T}$ cell-neuron interactions, cultured hippocampal neurons were treated for 48 hours with interferon- $\gamma(100 \mathrm{U} / \mathrm{ml})$ to enhance MHC-I expression levels, loaded with the OVA peptide and exposed to activated OVA-peptide-reactive OT-I T cells for 6 hours in culture (Figure 2, A and C). Incubation with GA alone caused apoptosis of cultured hippocampal neurons (fraction of activated caspase- $3^{-} \mathrm{NeuN}^{+}$neurons: GA $1 \mu \mathrm{g} / \mathrm{ml}: 73 \pm 12.5 \%$; GA $20 \mu \mathrm{g} / \mathrm{ml}: 76 \pm 15.7 \% ; n=$
A

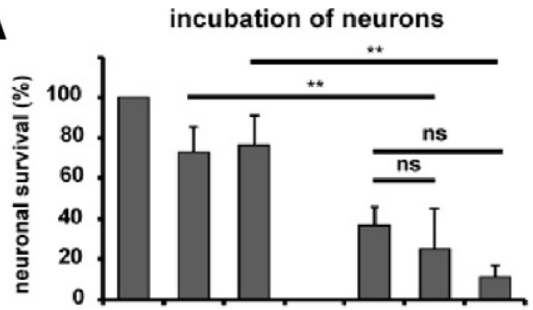

$\mathrm{GA}(\mu \mathrm{g} / \mathrm{ml}):-\quad 120$
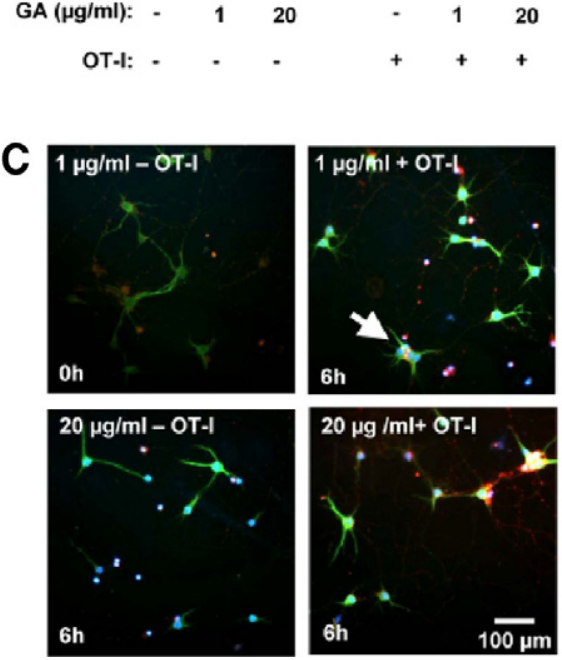

B incubation of OT-I T cells/mice
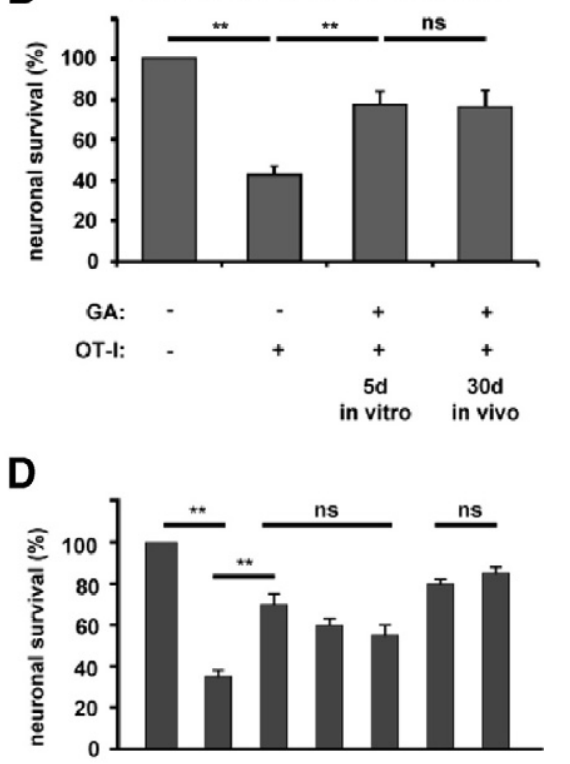

GA $(\mu \mathrm{g} / \mathrm{ml}): \begin{array}{lllllll}- & 0 & 0 & 1 & 20 & 1 & 20\end{array}$

OT-І: -+++++

OVA: - + - - - - -

preincubation of neurons

preincubation of OT-I
Figure 2. Apoptosis of OVA peptide-loaded MHC-I-expressing neurons induced by OVAspecific OT-I effector T cells is reduced by preincubation of OT-I splenocytes but not neurons with GA. A: Fractions of activated caspase- $3^{+}$ $\mathrm{NeuN}^{+}$cell bodies of OVA peptide-loaded hippocampal neurons in culture after 6 hours of incubation with and without in vitro activated OT-I T cells in the absence or presence of two concentrations of GA ( 1 and $20 \mu \mathrm{g} / \mathrm{ml}$ ). B: Fractions of activated caspase- $3^{+} \mathrm{NeuN}^{+}$cell bodies of OVA peptide-loaded hippocampal neurons in culture after 6 hours of incubation with and without activated OT-I T cells either pretreated with GA (1 or $20 \mu \mathrm{g} / \mathrm{ml})$ during antigen stimulation in vitro or isolated from OT-I mice pretreated with GA ( $100 \mathrm{mg} / \mathrm{kg} / \mathrm{d}$ i.p.) for 30 days. ns $=$ not significant, ${ }^{* *} P<0.05$. C: Representative immunofluorescence images of cultured hippocampal neurons incubated for 6 hours with and without in vitro activated OT-I T cells in the absence or presence of two concentrations of GA (1 and $20 \mu \mathrm{g} / \mathrm{ml})$. White arrow indicates an activated caspase $-3^{+} \mathrm{NeuN}^{+}$neuron DAPI (blue), NeuN (green), activated caspase-3 (red). Scale bar $=100 \mu \mathrm{m}$. D: Fractions of activated caspase- $3^{+} \mathrm{NeuN}^{+}$cell bodies of non-OVA peptide-loaded hippocampal neurons in culture after 6 hours of incubation with GA ( 1 and $20 \mu \mathrm{g} / \mathrm{ml}$ ) alone or with OT-I T cells activated in the absence or presence of GA (1 and $20 \mu \mathrm{g} / \mathrm{ml}$ ). ns $=$ not significant, ${ }^{* * *} P<0.05$. 
4 respectively). Exposition of OVA-peptide-loaded neurons to activated OT-I T cells reduced the fraction of nonapoptotic (caspase- $3^{-}$) $\mathrm{NeuN}^{+}$neurons to $36 \pm$ $9.5 \%$ after 6 hours, irrespective of the presence of $G A$ [GA $(1 \mu \mathrm{g} / \mathrm{ml}): 25 \pm 20 \%, P=0.29, n=4 ;$ GA $(20 \mu \mathrm{g} / \mathrm{ml})$ : $11 \pm 5.6 \%, P=0.41, n=4]$.

To test whether GA pretreatment of OT-I T cells could limit their capability of inducing neuronal apoptosis following direct interaction with OVA-antigen presenting neurons, OT-I T cells were incubated in the absence or presence of GA $(1,20$, and $100 \mu \mathrm{g} / \mathrm{ml})$ during 5 days of OVA-peptide stimulation in vitro. In a second in vivo approach, OT-I mice from which OT-I T cells were obtained were pretreated intraperitoneally (i.p.) with $\mathrm{GA}(100 \mathrm{mg} / \mathrm{kg} / \mathrm{d})$ for 30 days. GA dose-dependently reduced the proliferation of OVApeptide incubated OT-I splenocytes (Supplemental Figure S2, A and B, at http://ajp.amjpathol.org). However, it had no impact on the expression of the $\mathrm{T}$ cell surface activation markers CD11a, CD44, and CD62L on OT-I T cells analyzed after 5 days of OVA peptide stimulation (Supplemental Figure S2C at http://ajp.amjpathol.org). Again, neuronal cell death was determined by staining for activated caspase-3 after 6 hours of incubation with treated versus untreated OT-I T cells in culture. Exposition of OVApeptide-loaded neurons to untreated OT-I T cells reduced the fraction of nonapoptotic (caspase- $3^{-}$) neurons to $42.4 \pm 4.4 \%$ after 6 hours compared to control conditions $(P=0.001, n=4)$. In contrast, pretreatment of OT-I T cells on activation in vitro or OT-I mice in vivo largely prevented $\mathrm{CD8}^{+} \mathrm{T}$ cell-mediated neuronal apoptosis (fraction of activated caspase-3- $3^{-} \mathrm{NeuN}^{+}$neurons: GA treatment of OT-I T cells in vitro: $77.2 \pm 6.5 \%, P=0.0012, n=4$; GA treatment of OT-I mice in vivo: $75.9 \pm 8.27 \%, P=0.001, n=4$; Figure $2 \mathrm{~B})$.

\section{Pretreatment of OT-I Effector T Cells with GA Reduces Antigen-Dependent Neuronal Cytotoxicity Rather than Antigen-Independent Bystander Effects}

In our culture system, OT-I T cell-mediated neuronal killing likely consists of direct antigen-dependent cytotoxicity upon TCR-engagement and indirect antigen-independent bystander effects. To distinguish the potential GAeffect on both mechanisms, non-OVA peptide-loaded neurons were incubated for 6 hours in the absence or presence of GA with OT-I T cells, that were activated before either in the absence or the presence of GA (Figure 2D). In the absence of the OVA peptide-untreated OT-I T cells caused significant antigen-independent neuronal cell death (fraction of activated caspase- $3^{-} \mathrm{NeuN}^{+}$ neurons: $70 \pm 5 \% ; P=0.01 ; n=5$ ), which was about half of that observed in the presence of the OVA peptide $(35 \pm 3 \% ; n=5)$. In the presence of GA $(1 \mu \mathrm{g} / \mathrm{ml}$ and 20 $\mu \mathrm{g} / \mathrm{ml}$ ) even more neurons tended to die, although this effect was not statistically significant (fraction of activated caspase- $3^{-}$NeuN $^{+}$neurons: GA $1 \mu \mathrm{g} / \mathrm{ml}: 60 \pm 3 \%$, GA 20 $\mu \mathrm{g} / \mathrm{ml}: 55 \pm 5 \% ; P=0.31 ; n=5$ respectively). If OT-I T cells were activated in the presence of GA before co-incubation with non-OVA peptide-loaded neurons, antigen-indepen- dent neuronal cell death tended to be reduced, however this effect was also not statistically significant (fraction of activated caspase- $3^{-} \mathrm{NeuN}^{+}$neurons: GA $1 \mu \mathrm{g} / \mathrm{ml}: 80 \pm$ $2 \%$, GA $20 \mu \mathrm{g} / \mathrm{ml}: 85 \pm 3 \% ; P=0.19 ; n=5$, respectively).

Hence, the presence of GA neither reduced antigendependent $\mathrm{CD} 8^{+} \mathrm{T}$ cell-mediated neuronal killing nor antigen-independent bystander effects. Moreover, pretreatment of $\mathrm{CD}^{+} \mathrm{T}$ cells with $\mathrm{GA}$ reduces antigendependent neuronal cytotoxicity rather than antigen-independent bystander killing.

\section{GA Does Not Prevent Collateral Neuronal Cell Death in Acute Brain Slices from ODC-OVA Mice on Incubation for 6 Hours}

To dissect whether GA might prevent collateral neuronal apoptosis induced by $\mathrm{CD}^{+} \mathrm{T}$ cells directed against oligodendrocytes in intact CNS tissue, we incubated activated OVA peptide-reactive OT-I T cells in acute brain slices from ODC-OVA mice, which selectively express ovalbumin as a neo-self-antigen in oligodendrocytes under the control of a truncated myelin basic protein promotor. ${ }^{17,19}$ After 6 hours of incubation in the absence and presence of different GA concentrations (10 to $200 \mu \mathrm{g} /$ $\mathrm{ml}$ ) we stained for the density of activated caspase- $3^{+}$, $\mathrm{NeuN}^{+}$apoptotic neuronal cell bodies in two gray matter areas (hippocampus and cortex; Figure 3, A and C). Incubation of GA for 6 hours in ODC-OVA slices in the absence of OT-I T cells tended to increase the density of activated caspase $-3^{+}$neuronal cell bodies in both gray matter areas, although this tendency did not reach statistical significance [Figure 3, A and C, left panels; density of apoptotic neuronal cell bodies after 6 hours of incubation without OT-I T cells: Hippocampus: GA (0 $\mu \mathrm{g} / \mathrm{ml}): 10 \pm 2 / \mathrm{mm}^{2}$ vs. GA $(200 \mu \mathrm{g} / \mathrm{ml}): 15 \pm 3 / \mathrm{mm}^{2} ; P=$ $0.209 ; n=6$; Cortex: GA $(0 \mu \mathrm{g} / \mathrm{ml}): 11 \pm 1 / \mathrm{mm}^{2}$ vs. GA $\left.(200 \mu \mathrm{g} / \mathrm{ml}): 15 \pm 1 / \mathrm{mm}^{2} ; P=0.131 ; n=6\right]$. Moreover, GA did not affect the collateral neuronal apoptosis in both gray matter areas induced by activated oligodendrocytedirected OT-I T cells within the range of concentrations tested [Figure 3, A and C, middle panels; density of apoptotic neuronal cell bodies after 6 hours of incubation with OT-I T cells: Hippocampus (dentate gyrus, DG): GA $(0 \mu \mathrm{g} / \mathrm{ml}): 18 \pm 2 / \mathrm{mm}^{2}$ vs. GA $(200 \mu \mathrm{g} / \mathrm{ml}): 18 \pm 2 / \mathrm{mm}^{2}$; $P=0.698 ; n=5$; Cortex: GA $(0 \mu \mathrm{g} / \mathrm{ml}): 17 \pm 1 / \mathrm{mm}^{2}$ vs. GA $\left.(200 \mu \mathrm{g} / \mathrm{ml}): 18 \pm 2 / \mathrm{mm}^{2} ; P=0.718 ; n=5\right]$. Exemplary histological staining are shown for selected conditions (Figure 3, A and B). Notably, nuclei of activated caspase $-3^{+}$neurons show inhomogeneous DAPI staining, consistent with nuclear condensation and fragmentation due to apoptotic cell death. (Figure 3A, inset).

\section{Collateral Neuronal Apoptosis Can Be Reduced by Pre-Incubation of OT-I Splenocytes with GA before Incubation in Acute Brain Slices from ODC-OVA Mice}

Next we tested whether GA pretreatment of OT-I T cells during OVA-antigen activation in vitro could also reduce 
A

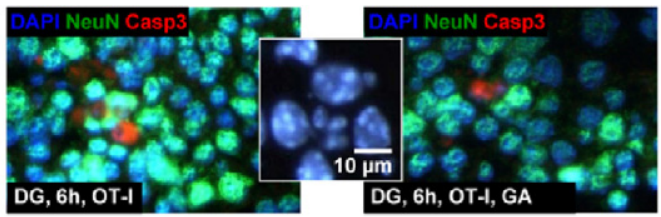

C

Hippocampus
OVA-ODC with OT-I

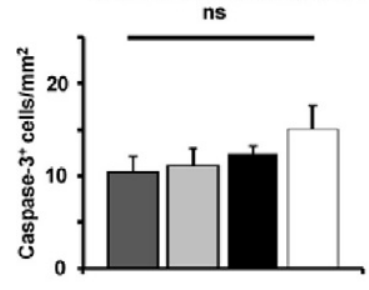

Cortex ns

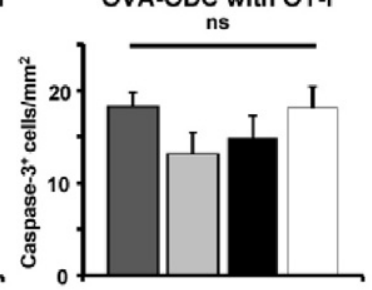

OVA-ODC without OT-I

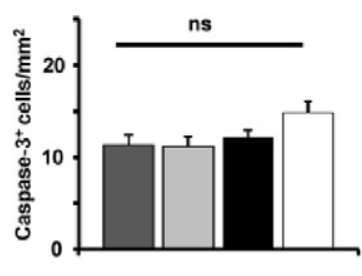

without GA
OVA-ODC with OT-I

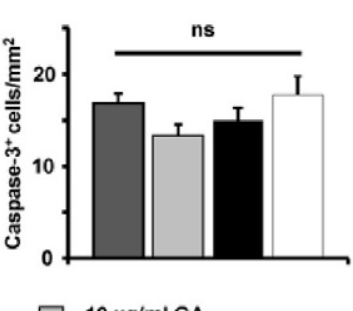

B

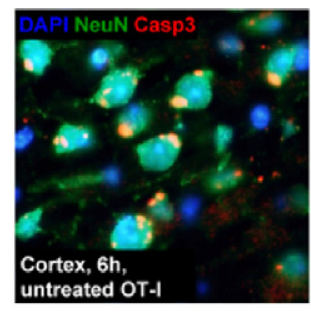

D

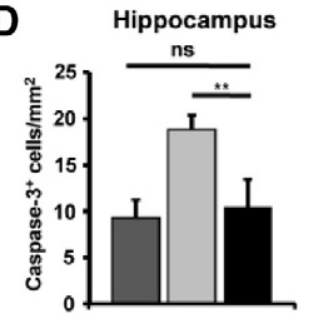

E

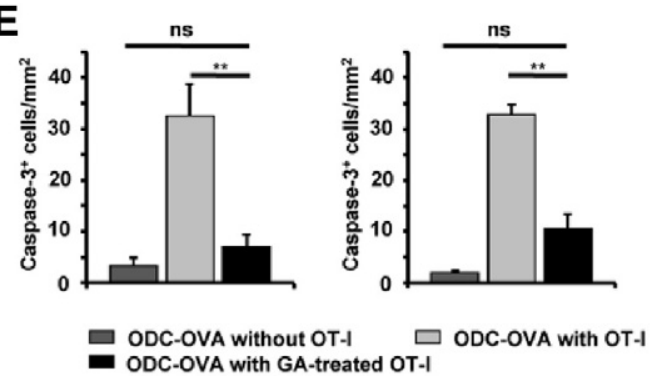

Figure 3. Direct oligodendroglial and collateral neuronal apoptosis in slices from ODC-OVA mice incubated with OVA-specific OT-I effector T cells is reduced by pre-incubation of OT-I splenocytes but not slices with GA. A and C: GA does not prevent neuronal cell death in acute brain slices from ODC-OVA mice on incubation for 6 hours with OT-I effector T cells. A: Representative immunofluorescence images of ODC-OVA slices incubated for 6 hours with in vitro activated OT-I T cells in the absence (left panel) and presence (right panel) of GA $(50 \mu \mathrm{g} / \mathrm{ml})$ in the hippocampus. (Inset) Nuclei of activated caspase- $3^{+}$neurons show inhomogeneous DAPI-staining consistent with nuclear condensation and fragmentation due to apoptotic cell death. DAPI (blue), NeuN (green), activated caspase-3 (red); scale bar represents $10 \mu \mathrm{m}$. C: Densities of activated caspase- $3^{+} \mathrm{NeuN}^{+}$neuronal cell bodies detected in the hippocampal (upper panels) and cortical (lower panels) gray matter of acute brain slices from ODC-OVA mice incubated for 6 hours with (right panels) and without (left panels) in vitro activated OT-I T cells in the absence and presence of three different GA concentrations (10, 50, and $200 \mu \mathrm{g} / \mathrm{ml})$. ns $=$ not significant, ${ }^{* *} P<0.05$. B, $\mathbf{D}$, and $\mathbf{E}$ : Neuronal (and oligodendroglial) apoptosis can be reduced by pre-incubation of OT-I splenocytes with GA before incubation in acute brain slices from ODC-OVA mice. B: Representative immunofluorescence images of ODC-OVA slices incubated for 6 hours with OT-I T cells in vitro activated in the absence (left panel) and presence (right panel) of GA $(1 \mu \mathrm{g} / \mathrm{ml})$. DAPI (blue), NeuN (green), activated caspase-3 (red). Scale bar $=10 \mu \mathrm{m}$. D: Densities of activated caspase- $3^{+}$NeuN ${ }^{+}$neuronal cell bodies detected in the hippocampal (left panel) and cortical (right panel) gray matter of acute brain slices from ODC-OVA mice incubated for 6 hours without (left bar) or with OT-I T cells in vitro activated in the absence (middle bar) and presence (right bar) of GA ( $1 \mu \mathrm{g} / \mathrm{ml})$. E: Densities of activated caspase- $3^{+}$NogoA ${ }^{+}$ oligodendrocytes detected in the hippocampal (left panel) and cortical (right panel) gray matter of acute brain slices from ODC-OVA mice incubated for 6 hours without (left bar) or with OT-I T cells in vitro activated in the absence (middle bar) and presence (right bar) of $\mathrm{GA}(1 \mu \mathrm{g} / \mathrm{ml}) . \mathrm{ns}=\mathrm{not}$ significant, ${ }^{*} P<0.05$.

collateral neuronal apoptosis in ODC-OVA slices ex vivo (consistent with our findings in cultured OVA-peptideloaded hippocampal neurons), which was shown to be strictly antigen-dependent. ${ }^{19}$ OT-I T cells activated in the absence or presence of GA $(1,20$, and $100 \mu \mathrm{g} / \mathrm{ml})$ were incubated for 6 hours in ODC-OVA slices, and subsequently the density of activated caspase $-3^{+}$neuronal cell bodies in the cortex and hippocampus was determined (Figure 3 B). In both gray matter areas, pretreatment of OT-I T cells reduced the density of activated caspase $-3^{+}$neuronal cell bodies to background levels [Figure 3D; density of apoptotic neuronal cell bodies after 6 hours of incubation (Hippocampus: without OT-I T cells: $9 \pm 2 / \mathrm{mm}^{2}$; with untreated OT-I T cells: $19 \pm 2 / \mathrm{mm}^{2} ; P=$ 0.004 ; with GA pretreated OT-I T cells: $10 \pm 3 / \mathrm{mm}^{2} ; P=$ 0.04; $n=5$ respectively; Cortex: without OT-I T cells: $11 \pm 1 / \mathrm{mm}^{2}$; with untreated OT-I T cells: $19 \pm 2 / \mathrm{mm}^{2} ; P=$ 0.021 ; with GA pretreated OT-I T cells: $12 \pm 1 / \mathrm{mm}^{2} ; P=$ $0.048 ; n=5$ respectively].

Consistently, pretreatment of OT-I T cells also significantly reduced the density of activated caspase $-3^{+}$oli- godendrocytes as the primary target cells of OT-I T cells in both gray matter areas [Figure 3E; density of apoptotic oligodendrocytes after 6 hours of incubation (Hippocampus: without OT-I T cells: $3 \pm 2 / \mathrm{mm}^{2}$; with untreated OT-I T cells: $32 \pm 2 / \mathrm{mm}^{2} ; P=0.004$; with GA pretreated OT-I T cells: $7 \pm 2 / \mathrm{mm}^{2} ; P=0.014 ; n=5$, respectively; Cortex: without OT-I T cells: $2 \pm 1 / \mathrm{mm}^{2}$; with untreated OT-I T cells: $33 \pm 2 / \mathrm{mm}^{2} ; P=0.0002$; with GA pretreated OT-I T cells: $11 \pm 3 / \mathrm{mm}^{2} ; P=0.0006 ; n=5$, respectively)].

Hence, GA-pretreatment of OT-I T cell reduced antigen-dependent direct and collateral neuronal cell death in our co-culture and slice model.

\section{In Focal EAE, Lesion Size and Neuronal Apoptosis Could Be Limited by Pretreating Rats but Not by I.C. Application of GA into the Inflammatory Lesion}

Thus far, our data strongly argue against a direct effect of GA on neuronal survival under inflammatory conditions. In contrast, the view of a largely reduced cytotoxic ca- 

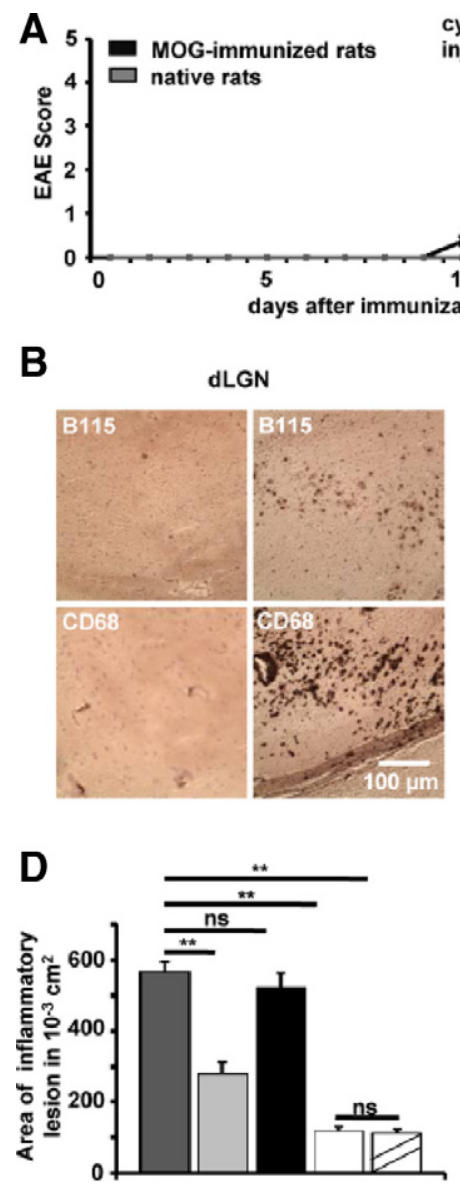

C
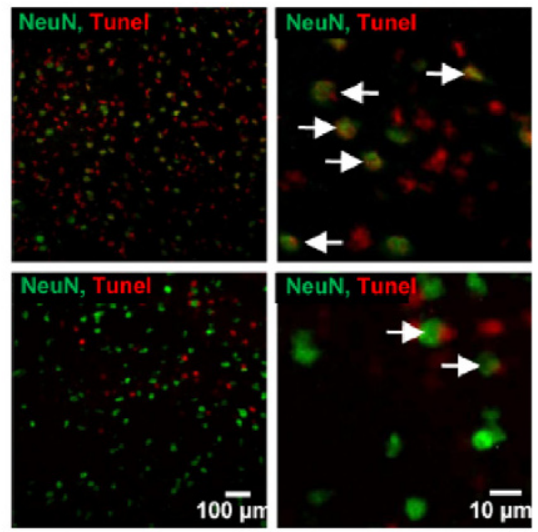

E

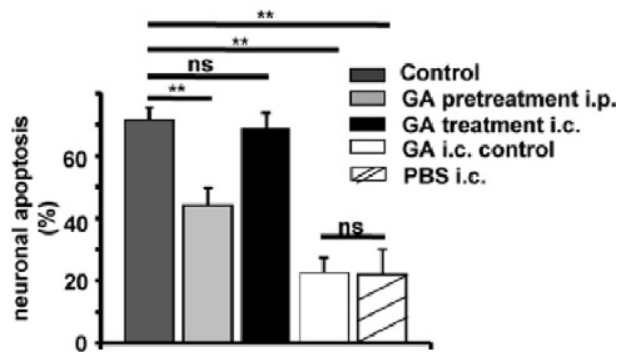

Figure 4. In focal EAE, lesion size and neurona apoptosis can be limited by pretreating rats, but not by i.c. application of GA into the inflammatory lesion. A: Clinical course of MOG-induced EAE in female DA rats. B: Histological analysis of the dLGN of MOG-immunized mice, at the side of i.c cytokine injection (right panels) and the contralateral control side (left panels). Stainings are shown for T cells (B115, upper panels) and macrophages (CD68, lower panels). C: Representative immunofluorescence images within the dLGN of MOG-immunized rats without (upper panel) and with GA pretreatment for 30 days (lower panel). NeuN (green), TUNEL (red), and TUNEL ${ }^{+}$ neuronal cell bodies are indicated by white arrows. Scale bar $=100$ or $10 \mu \mathrm{m}$. D and E: Quantitative analysis of lesion size and fraction of NeuN $\mathrm{TUNEL}^{+}$neurons within the dLGN in MOG-immunized rats 2 days after i.c. injection of pro-inflammatory cytokines (control), in MOG-immunized rats i.p. treated with $\mathrm{GA}[2 \mathrm{mg} / \mathrm{d}$, GA pretreatment (30 days)], in MOG-immunized rats that received i.c. injection of pro-inflammatory cytokines with GA ( $40 \mu \mathrm{g}$; GA treatment i.c.) and immunized rats that received a control injection with GA ( $40 \mu \mathrm{g}$, GA i.c. control) or PBS alone. ns = not significant ${ }^{* * *} P<0.05$ pacity of $\mathrm{CD}^{+} \mathrm{T}$ cells activated in the presence of GA is supported. To finally prove the concept of an indirect prevention of neuronal cell death by GA-induced modulation of the adaptive immune response, we studied the impact of peripheral GA treatment versus i.c. GA injection into the inflammatory lesion on neuronal apoptosis in a focal EAE model. ${ }^{25}$

Focal lesions were induced 24 hours after the onset of clinical symptoms following MOG immunization by cytokine injection to the region of interest (dLGN, Figure 4A). Two days after cytokine injection, histological analysis of the dLGN at the side of injection (Figure 4B, right panels) and the contralateral control side (Figure 4B, left panels) was performed. Cellular infiltrates were analyzed using the pan-T cell marker B115 (Figure 4B, upper panels) and CD68 (Figure 4B, lower panels) for macrophage/ microglia labeling. Cell infiltration could only be observed within the inflamed dLGN, but not on the contralateral side. As controls, we stained for B115 in the dLGN of immunized rats that received i.c. injection with saline and nonimmunized rats that were injected with cytokines. Under both conditions only individual $\mathrm{B}_{115^{+}} \mathrm{T}$ cells could be detected in the injected dLGN, which did not significantly differ from the noninjected control side in immunized rats.

To test the impact of direct versus indirect effects of GA on gray matter lesion pathology in the focal EAE model, rats were either i.p. injected with GA for 30 days
(100 mg/kg/d) before and after MOG immunization (pretreatment) or were injected i.c. with $2 \mu \mathrm{l} \mathrm{GA}(20 \mu \mathrm{g} / \mathrm{ml})$ at the site of focal lesion formation at the onset of clinical symptoms. Two days after injection, rats were sacrificed and the dLGN was analyzed for lesion size (by staining for $\mathrm{CD} 8^{+}$macrophages/microglia) and the fraction of apoptotic neurons within the lesion (by staining for TUNEL $^{+} \mathrm{NeuN}^{+}$neuronal cell bodies, Figure 4C). Those rats, in which focal gray matter inflammation was induced via MOG immunization and i.c. cytokine injection exhibited a dLGN lesion size of $0.57 \pm 0.03 \mathrm{~cm}^{2}(n=6)$ and a fraction of $\mathrm{TUNEL}^{+}$apoptotic $\mathrm{NeuN}^{+}$neuronal cell bodies of $72 \pm 4 \%$ (Figure 4 , D and $\mathrm{E} ; n=6$ ). Pretreatment of rats for 30 days before and after MOG-immunization significantly reduced both the lesion size (0.28 \pm $\left.0.03 \mathrm{~cm}^{2}, P=0.0001, n=6\right)$ and the fraction of apoptotic neurons (44 $\pm 6, P=0.01, n=6$ ) within the dLGN. In contrast, direct i.c. injection of $\mathrm{GA}$ into the region of interest had no impact on lesion size $\left(0.52 \pm 0.04 \mathrm{~cm}^{2}\right.$, $P=0.434, n=6)$ and the extent of neuronal apoptosis $(69 \pm 5 \%, P=0.728, n=6)$. The i.c. GA injection into the dLGN of immunized and non-cytokine injected rats elicited a lesion size of $0.12 \pm 0.01 \mathrm{~cm}^{2}(n=6)$ with a fraction of apoptotic neurons of $22 \pm 5 \%$, $(P=0.0001$, $n=6)$. This level was comparable to control conditions (PBS i.c., lesion: $0.12 \pm 0.01 \mathrm{~cm}^{2}$, fraction of apoptotic neurons: $21 \pm 6 \%$ ). Taken together our results which combine a single cell- and a network-based in vitro, a 
slice-based ex vivo, as well as an in vivo approach indicate that GA attenuates the adaptive $\mathrm{T}$ cell response but does not exert any direct neuroprotective effect.

\section{Discussion}

Recently, it was suggested that GA is able to permeate the (impaired) blood-brain barrier and directly protect neurons/axons under conditions of inflammation-related neurodegeneration. ${ }^{15}$ Hence, we here examined direct effects of GA on electrical properties of neurons and T cell-mediated neuronal apoptosis using different in vitro, ex vivo, and in vivo models. We show that GA attenuates the pro-inflammatory adaptive $T$ cell response and thereby limits inflammatory neuronal cell death. However, direct interaction of GA with neurons is deleterious: Single cell patch clamp recordings showed that within 10 minutes, GA caused a depolarization of the resting membrane potential and an immediate impairment of action potential generation in cultured hippocampal neurons independently from its binding to MHC I molecules. This caused an immediate reversible increase in neuronal network activity as assessed by multi-electrode recording of compound field potential in cultured hippocampal neurons. Moreover, GA incubated hippocampal neurons underwent dose-dependent apoptosis within 6 hours in culture. Apoptosis of OVA peptide-loaded MHC1 -expressing neurons induced by OVA-specific OT-I effector $T$ cells could be reduced by pre-incubating OT-I splenocytes but not neurons with GA. Similarly, in acute brain slices from ODC-OVA mice, incubation of GA tended to increase the density of apoptotic neurons in gray matter regions and did not prevent neuronal cell death on incubation for 6 hours with OT-I effector T cells. However, collateral neuronal (and direct oligodendroglial) apoptosis could be reduced by pre-incubation of OT-I splenocytes with GA. In focal EAE, lesion size and neuronal apoptosis could be limited by pretreating rats with GA for 30 days. Importantly, i.c. application of GA into the inflammatory lesion circumventing the blood-brain barrier also had no effect on the fraction of apoptotic neurons in the gray matter lesion. We therefore conclude that GA modulates the adaptive $T$ cell response, but does not directly protect neurons form inflammatory cell death. In contrast, prolonged direct exposition of neurons with GA per se in culture, in acute brain slices and within the gray matter in focal EAE caused apoptotic cell death. This is consistent with the depolarization of the neuronal membrane potential and the cessation of action potential generation observed immediately in neurons exposed to GA. Depolarization of the membrane potential is likely to activate several voltage- and ligand-gated cation conductance in neurons exposed to GA and cause cation overload and subsequent cell death. At the moment however we cannot delineate the exact molecular mechanism of GA-induced neuronal cell death.

Neuronal cell death during direct antigen-dependent $\mathrm{CD}^{+} \mathrm{T}$ cell-neuron interaction ${ }^{22}$ and collateral neuronal apoptosis during an ODC-directed $\mathrm{CD} 8^{+} \mathrm{T}$ cell attack in acute brain slices ${ }^{19}$ could be limited either by pretreatment of $\mathrm{T}$ cell donor mice in vivo or by co-incubation during antigen-stimulation in vitro. This is consistent with the notion that GA treatment is capable of limiting antigen-dependent pro-inflammatory $\mathrm{CD} 8^{+} \mathrm{T}$ cell activation. Moreover, inflammatory neuronal cell death could be reduced in MOG-induced focal EAE ${ }^{25}$ (predominantly mediated by myelin-directed $\mathrm{CD} 4^{+} \mathrm{T}$ cells) by GA pretreatment of rats for 30 days. In turn this is consistent with the notion that GA is capable of attenuating pro-inflammatory $\mathrm{CD}^{+} \mathrm{T}$ cell responses. ${ }^{9,10}$ In line with the recent finding that GA modulates the adaptive $T$ cell response independently from its binding to MHC II molecules on APCs, ${ }^{4}$ we found that the deleterious effects of GA on neurons occur independently from its binding to neuronal MHC I molecules.

In conclusion, our results strongly argue against a direct neuroprotective mechanism of GA. In contrast they suggest that different parts of the adaptive $T$ cell response (here $\mathrm{CD}^{+}$and $\mathrm{CD} 8^{+}$) can be modulated by GA, thereby leading to a milder immune response under (auto)inflammatory conditions.

\section{Acknowledgments}

We thank Svetlana Hilz, Astrid Schmitt, and Andrea Sauer for excellent technical assistance.

\section{References}

1. Duda PW, Schmied MC, Cook SL, Krieger JI, Hafler DA: Glatiramer acetate (Copaxone) induces degenerate. Th2-polarized immune responses in patients with multiple sclerosis. J Clin Invest 2000, 105:967-9762

2. Fridkis-Hareli M, Teitelbaum D, Gurevich E, Pecht I, Brautbar C, Kwon OJ, Brenner T, Arnon R, Sela M: Direct binding of myelin basic protein and synthetic copolymer 1 to class II major histocompatibility complex molecules on living antigen-presenting cells-specificity and promiscuity. Proc Natl Acad Sci USA 1994, 91:4872-4876

3. Neuhaus O, Farina C, Yassouridis A, Wiendl H, Then Bergh F, Dose T, Wekerle H, Hohlfeld R: Multiple sclerosis: comparison of copolymer-1- reactive $T$ cell lines from treated and untreated subjects reveals cytokine shift from T helper 1 to T helper 2 cells. Proc Natl Acad Sci USA 2000, 97:7452-7457

4. Weber MS, Prod'homme T, Youssef S, Dunn SE, Rundle CD, Lee L, Patarroyo JC, Stuve O, Sobel RA, Steinman L, Zamvil SS: Type II monocytes modulate $T$ cell-mediated central nervous system autoimmune disease. Nat Med 2007, 13:935-943

5. Aharoni R, Eilam R, Domev H, Labunskay G, Sela M, Arnon R: The immunomodulator glatiramer acetate augments the expression of neurotrophic factors in brains of experimental autoimmune encephalomyelitis mice. Proc Natl Acad Sci USA 2005, 102:19045-19050

6. Gorantla S, Liu J, Sneller H, Dou H, Holguin A, Smith L, Ikezu T, Volsky DJ, Poluektova L, Gendelman HE: Copolymer-1 induces adaptive immune anti-inflammatory glial and neuroprotective responses in a murine model of HIV-1 encephalitis. J Immunol 2007, 179:4345-4356

7. Maier K, Kuhnert AV, Taheri N, Sattler MB, Storch MK, Williams SK, Bahr M, Diem R: Effects of glatiramer acetate and interferon-beta on neurodegeneration in a model of multiple sclerosis: a comparative study. Am J Pathol 2006, 169:1353-1364

8. Benner EJ, Mosley RL, Destache CJ, Lewis TB, Jackson-Lewis V, Gorantla S, Nemachek C, Green SR, Przedborski S, Gendelman HE: Therapeutic immunization protects dopaminergic neurons in a mouse model of Parkinson's disease. Proc Natl Acad Sci USA 2004, 101: 9435-9440

9. Kipnis J, Yoles E, Porat Z, Cohen A, Mor F, Sela M, Cohen IR, 
Schwartz M: T cell immunity to copolymer 1 confers neuroprotection on the damaged optic nerve: possible therapy for optic neuropathies. Proc Natl Acad Sci USA 2000, 97:7446-7451

10. Schori H, Kipnis J, Yoles E, WoldeMussie E, Ruiz G, Wheeler LA, Schwartz M: Vaccination for protection of retinal ganglion cells against death from glutamate cytotoxicity and ocular hypertension: implications for glaucoma. Proc Natl Acad Sci USA 2001, 98: 3398-3403

11. Aharoni R, Kayhan B, Eilam R, Sela M, Arnon R: Glatiramer acetatespecific $T$ cells in the brain express $T$ helper $2 / 3$ cytokines and brain-derived neurotrophic factor in situ. Proc Natl Acad Sci USA 2003, 100:14157-14162

12. Aharoni R, Teitelbaum D, Leitner O, Meshorer A, Sela M, Arnon R: Specific Th2 cells accumulate in the central nervous system of mice protected against experimental autoimmune encephalomyelitis by copolymer 1. Proc Natl Acad Sci USA 2000, 97:11472-11477

13. Shaked I, Tchoresh D, Gersner R, Meiri G, Mordechai S, Xiao X, Hart RP, Schwartz M: Protective autoimmunity: interferon-gamma enables microglia to remove glutamate without evoking inflammatory mediators. J Neurochem 2005, 92:997-1009

14. Aharoni R, Arnon R, Eilam R: Neurogenesis and neuroprotection induced by peripheral immunomodulatory treatment of experimental autoimmune encephalomyelitis. J Neurosci 2005, 25:8217-8228

15. Liu J, Johnson TV, Lin J, Ramirez SH, Bronich TK, Caplan S, Persidsky $Y$, Gendelman HE, Kipnis J: T cell independent mechanism for copolymer-1-induced neuroprotection. Eur J Immunol 2007, 37: 3143-3154

16. Linker RA, Maurer M, Gaupp S, Martini R, Holtmann B, Giess R, Rieckmann P, Lassmann H, Toyka KV, Sendtner M, Gold R: CNTF is a major protective factor in demyelinating CNS disease: a neurotrophic cytokine as modulator in neuroinflammation. Nat Med 2002, 8:620-624

17. Cao Y, Toben C, Na SY, Stark K, Nitschke L, Peterson A, Gold R, Schimpl A, Hunig T: Induction of experimental autoimmune encephalomyelitis in transgenic mice expressing ovalbumin in oligodendrocytes. Eur J Immunol 2006, 36:207-215

18. Hogquist KA, Jameson SC, Heath WR, Howard JL, Bevan MJ, Car- bone FR: T cell receptor antagonist peptides induce positive selection. Cell 1994, 76:17-27

19. Gobel K, Melzer N, Herrmann AM, Schuhmann MK, Bittner S, Ip CW, Hunig T, Meuth SG, Wiendl H: Collateral neuronal apoptosis in CNS gray matter during an oligodendrocyte-directed CD8(+) T cell attack. Glia 2009, 58:469-480

20. Leder C, Ortler S, Seggewiss R, Einsele H, Wiendl H: Modulation of T-effector function by imatinib at the level of cytokine secretion. Exp Hematol 2007, 35:1266-1271

21. Lafon M, Megret F, Meuth SG, Simon O, Velandia Romero ML, Lafage M, Chen L, Alexopoulou L, Flavell RA, Prehaud C, Wiendl H: Detrimental contribution of the immuno-inhibitor $\mathrm{B} 7-\mathrm{H} 1$ to rabies virus encephalitis. J Immunol 2008, 180:7506-7515

22. Meuth SG, Herrmann AM, Simon OJ, Siffrin V, Melzer N, Bittner S, Meuth P, Langer HF, Hallermann S, Boldakowa N, Herz J, Munsch T, Landgraf $\mathrm{P}$, Aktas $\mathrm{O}$, Heckmann M, Lessmann V, Budde T, Kieseier $\mathrm{BC}$, Zipp F, Wiendl H: Cytotoxic CD8+ T cell-neuron interactions: perforin-dependent electrical silencing precedes but is not causally linked to neuronal cell death. J Neurosci 2009, 29:15397-15409

23. Illes S, Fleischer W, Siebler M, Hartung HP, Dihne M: Development and pharmacological modulation of embryonic stem cell-derived neuronal network activity. Exp Neurol 2007, 207:171-176

24. Edwards FA, Konnerth A, Sakmann B, Takahashi T: A thin slice preparation for patch clamp recordings from neurones of the mammalian central nervous system. Pflugers Arch 1989, 414:600-612

25. Merkler D, Ernsting T, Kerschensteiner M, Bruck W, Stadelmann C: A new focal EAE model of cortical demyelination: multiple sclerosis-like lesions with rapid resolution of inflammation and extensive remyelination. Brain 2006, 129:1972-1983

26. Adelmann M, Wood J, Benzel I, Fiori P, Lassmann H, Matthieu JM, Gardinier MV, Dornmair K, Linington C: The N-terminal domain of the myelin oligodendrocyte glycoprotein (MOG) induces acute demyelinating experimental autoimmune encephalomyelitis in the Lewis rat. J Neuroimmunol 1995, 63:17-27

27. Dixon W, Massey FJ: Introduction to statistical analysis. New York, McGraw-Hill Companies, 1969 